procedural period. In observational cohorts and one randomised study (PIONEER-AF), uninterrupted NOAC therapy has also been found to be safe in patients undergoing AF ablations. EHRA states that simple ablation procedures have a low bleeding risk while complex ablation procedures have high bleeding risk. We describe our experience of a wide variety of catheter ablations performed on uninterrupted NOAC therapy and compare our results with similar procedures undertaken on uninterrupted warfarin therapy.

Methods A retrospective analysis of the NICOR database for all ablations undertaken on uninterrupted anticoagulation at the New Cross Hospital, Wolverhampton, between April 2014 and August 2016 was undertaken. Data regarding the number and type of procedures, type of oral anticoagulation (warfarin versus NOACs), frequency of trans-septal punctures, DCCV during procedures and complications were analysed.

Results Atrial of 648 ablations were performed in the study period of which328(50.7\%) were undertaken on uninterrupted anticoagulants (uninterrupted warfarin group (uW Grp): 228 (35.1\%) and uninterrupted NOAC group (UNOAC Grp): 101 (15.5\%). Mean age was 59 in both groups with more male preponderance in the NOAC group. A range of simple and complex ablations were done including 131 (57.4\%) AF in UW Grp compared to $26(26.5 \%)$ in the uNOAC grp. Transseptal punctures were more common in the UW Grp compared to the uNOAC Grp, however cardioversions (both external and internal) were used in similar fashion. Composite of bleeding and thrombo-embolic complications were relatively low in both the groups $[n=5(2.1 \%)$ in the VKA group compared to $n=1(0.9 \%)$ in the UNOAC Grp with OR 2.21 (95\% CI 0.25 to $19.2 ; \mathrm{p}=0.47)]$.

Conclusions Our experience suggests that a wide range of simple and complex ablation procedures can be safely performed ion uninterrupted NOAC therapy. Complications in the uNOAC Grp were lower than the uW Grp, reflecting growing confidence among electrophysiologists to undertake such procedures even in the absence of antidote for majority of the NOACs.

\begin{tabular}{lll} 
Abstract & $\mathbf{4 7}$ & Table 1 \\
\hline Variables & $\begin{array}{l}\text { Uninterrupted Warfarin group } \\
(\mathbf{n}=228)\end{array}$ & $\begin{array}{l}\text { uninterrupted NOAC group } \\
(\mathbf{n}=101)\end{array}$ \\
\hline Mean Age & 59 & 59 \\
Male & $59 \%$ & $67 \%$ \\
Mean & 2.02 & 2.1 \\
CHA2DS2VAsc & & \\
Simple ablations & 90 & 76 \\
Complex & 139 & 27 \\
ablations & & \\
Trans septals & 149 & 27 \\
DCCV & 67 & 23 \\
Complications & 10 & 2 \\
\hline
\end{tabular}

\section{UTILISATION OF CARDIAC IMPLANTABLE ELECTRONIC DEVICE THERAPY IN PATIENTSWITH ATTR-WILD TYPE AMYLOIDOSIS WITH CONCURRENT CARDIAC INVOLVEMENT}

Anwar A Chahal* ${ }^{*}$ Deepak Padmanabhan, Nidhi Tandon, Virend K Somers, Angela Dispenzieri, Martha Grogan, Peter A Brady, Grace Lin. Mayo Clinic

\subsection{6/heartjnl-2017-311726.47}

Introduction The role of cardiac implantable electronic device (CIED) therapy in patients with ATTR-wt (senile) amyloid is not very well defined. It is unknown whether there is mortality benefit or harm with the implantation of CIEDs.

Methods We performed a retrospective analysis of a prospectively collected registry of patients with biopsy proven ATTRwt amyloid with cardiac involvement. These patients were followed up at Mayo Clinic, Rochester, Minnesota between Jan 1, 1985 to Sep 30, 2015. The follow-up of these patients was done via personal communication (written or verbal) and entered into the database. During analysis these patients were subdivided on the basis of the presence or absence of a CIED, cardiac involvement or not, and all-cause mortality analysed. The implantation of a CIED was analysed as a timedependent covariate in the survival of these patients and Kaplan-Meier (K-M) survival plots created. Comparison between groups with CIED and those without CIED was made. In addition, CIEDs were subdivided into permanent pacemakers (PPM), defibrillators (ICDs) and cardiac resynchronization devices (CRT-P/D). Mortality among the patients with each device subtype was estimated and compared to overall mortality.

Results 409 patients with ATTR-wt and cardiac involvement were reviewed with CIEDs implanted in 101 (25\%) patients. The median follow-up (25th, 75th centile) was 2.4 (1.1, 4.3) years. Utilisation of ICDs in this population was higher than the general community in Olmsted county. There was no association in the overall survival or evidence of harm with CIED utilisation in patients with ATTR amyloidosis and cardiac involvement.

Conclusions CIEDs utilisation remains high in ATTR-wt with cardiac involvement. Due diligence is needed in selecting the optimal patient for benefit prior to the implantation of CIEDs in this cohort. Further research is required to pool multicentre amyloid registry data and determine if there is mortality benefit.

\section{PREDICTING RISK OF SCD IN FABRY DISEASE: A SINGLE CENTRE EXPERIENCE}

${ }^{1}$ Shanat Baig*, ${ }^{1}$ Nicky Edwards, ${ }^{2}$ Sabrina Nordin, ${ }^{1}$ Boyang Liu, ${ }^{2}$ James Moon, ${ }^{1}$ Tarek Geberhiwot, ${ }^{1}$ Richard P Steeds. 'Queen Elizabeth Hospital; ${ }^{2}$ Barts Heart Centre

10.1136/heartjnl-2017-311726.48

Introduction Fabry disease (FD) is a rare X-linked lysosomal storage disorder with a variable cardiac phenotype and a 